\title{
The protective effects of Ocimum basilicum extract against gentamicin-induced nephrotoxicity in male rats; an anti-inflammatory, anti-oxidative and anti- apoptotic action
}

\author{
Hassan Askari $^{1}$, Fatemeh Zeinali², Hamed Haghi-Aminjan ${ }^{3}$, Seyed Mehdi Hafizi ${ }^{4}$, Amirhesam Alirezaei ${ }^{5^{*}}$ \\ ${ }^{1}$ Transplantation Research Center, Shariati Hospital, Tehran University of Medical Sciences, Tehran, Iran \\ ${ }^{2}$ Department of Biology, Tehran University, Tehran, Iran \\ ${ }^{3}$ Drug and Advanced Sciences Research Center, Ardabil University of Medical Sciences, Ardabil, Iran \\ ${ }^{4}$ Department of Internal Medicine, Shahid Beheshti University of Medical Sciences, Tehran, Iran \\ ${ }^{5}$ Clinical Research and Development Center, Shahid Modarres Hospital, Department of Nephrology, Shahid Beheshti University \\ of Medical Sciences, Tehran, Iran
}

\section{*Correspondence to \\ Amirhesam Alirezaei, Email: \\ Amirhesam124@gmail.com}

Received 20 March 2019

Accepted 11 May 2019

Published online 15 June 2019

Keywords: Acute kidney injury, Apoptosis, Gentamicin, Nephrotoxicity, Ocimum basilicum Herb, Traditional medicine, Renal failure, Oxidative stress

\section{Abstract}

Introduction: Some studies are in favor of protective and anti-inflammatory effects of Ocimum basilicum (OB) against renal failure.

Objectives: The aim of this study was to explore the protective effects of OB extract against gentamicin (GM)-induced nephrotoxicity in male rat models of acute kidney injury.

Materials and Methods: Twenty-seven adult male Wistar rats with the weight of 200-250 g were prepared under standard condition ( $12 \mathrm{~h} \mathrm{light/dark} \mathrm{cycle,} 20$ to $22^{\circ} \mathrm{C}$ temperature). In the gentamicin group (GM), the rats were administrated normal saline (5 mL/kg; oral) for four consecutive weeks and then $\mathrm{GM}(80 \mathrm{mg} / \mathrm{kg} / \mathrm{d}$; intraperitoneal) for another one week. In the third (OB-200+ GM) and fourth (OB-800+ GM) groups, the rats were initially treated with OB extract (200 $\mathrm{mg} / \mathrm{kg}$ and $800 \mathrm{mg} / \mathrm{kg}$ respectively; oral) for four consecutive weeks followed by intraperitoneal administration of $80 \mathrm{mg} / \mathrm{kg} / \mathrm{d}$ GM for another one week. The control group was treated with normal saline $(5 \mathrm{~mL} / \mathrm{kg}$; oral) for five consecutive weeks. They were sacrificed under deep anesthesia, then plasma and tissue samples were obtained.

Results: Treatment with OB hydro-alcoholic extract decreased BUN, serum $\mathrm{Cr}$, FeNa and the levels of TNF-a and NF-kB and also diminished the rate of apoptosis in renal tubular cells compared to gentamicin group. Conclusion: Our findings provide comprehensive evidence supporting the beneficial role of $\mathrm{OB}$, a traditional medicinal herb, as a potential therapeutic supplement to prevent the incidence and progression of renal impairment in GM-induced renal toxicity.

Citation: Askari H, Zeinali F, Haghi-Aminjan $\mathrm{H}$, Hafizi SM, Alirezaei A The protective effects of Ocimum basilicum extract against gentamicin-induced nephrotoxicity in male rats; an anti-inflammatory, antioxidative and anti-apoptotic action. Immunopathol Persa. 2019;5(2):e21. DOI: 10.15171 ipp.2019.21

\section{Introduction}

Acute kidney injury (AKI) is defined as renal dysfunction secondary to acute cellular and structural damages to renal tissues. As kidneys have a key role in the proper function of several organs, AKI can manifest as a clinical syndrome with multiorgan dysfunctions (1-2). Toxin-induced nephrotoxicity (TIN) is one of the main reasons of AKI in the general population. It has been reported that $10 \%-20 \%$ of pharmacological agents can induce toxininduced nephrotoxicity $(1,2)$.

Gentamicin (GM) is a well-known aminoglycoside antibiotic inhibiting the growth of gram-negative microorganisms

\section{Key point}

In an experimental study we observed that Ocimum basilicum extract induced anti-apoptotic effects on renal tubular cells and also ameliorated gentamicin induced nephrotoxicity in male rats.

(3). The administration of GM in clinical practice is restricted due to its nephrotoxicity and ototoxicity (3). If the duration of GM administration exceeds from 14 days, the incidence of GM-induced nephrotoxicity increases by approximately 50\% (4-6). Histologically, GM-induced nephrotoxicity is characterized by tubular necrosis, basement membrane destruction, and

\footnotetext{
Copyright $\left({ }_{0} 2019\right.$ The Author(s); Published by Nickan Research Institute. This is an open-access article distributed under the terms of the Creative Commons Attribution License (http://creativecommons.org/licenses/by/4.0), which permits unrestricted use, distribution, and reproduction in any medium, provided the original work is properly cited.
} 
cellular apoptosis leading to reduced glomerular filtration and renal dysfunction (4-6).

GM reduces the level of cellular ATP by inhibiting the phosphorylation process in renal tubular cells. On the other hand, GM can induce inflammation, oxidation and apoptosis by increasing the expressions of TNF-receptor type1 (TNF-R1), inducible nitric oxide synthase (iNOS), COX-2 inhibitor, and nuclear factor kappa-light-chainenhancer of activated B cells (NF- $\mathrm{BB}$ ). Furthermore, GM decreases the expression of B-cell lymphoma-extra-large (Bcl-XL) anti-apoptotic gene (5-7).

Ocimum basilicum (OB) is a plant growing in Iran and several countries. $\mathrm{OB}$ has commonly been applied as an herbal remedy in traditional medicine. Several investigations have described the beneficial roles of $\mathrm{OB}$ in lowering blood glucose and lipids, accelerating wound healing, scavenging free radicals and inhibiting the growth of microorganisms $(8,9)$. This herbal drug has also been utilized for treating insomnia, abdominal cramps, migraines, gastroenteritis, diarrhea and malabsorption. There are no reports of toxicity related to this plant $(10,11)$. The nephroprotective activity of $\mathrm{OB}$ extract has been reported in some studies $(10,11)$. However, the current knowledge on the possible nephroprotective mechanisms of $\mathrm{OB}$ is inadequate. In addition, the protective role of $\mathrm{OB}$ extract against GM-induced nephrotoxicity remains unclear.

\section{Objectives}

The aim of this study was to explore the protective effects of hydro-alcoholic extract of $\mathrm{OB}$ against GM-induced nephrotoxicity in male rats.

Materials and Methods

Experimental animals

Twenty-seven adult male Wistar rats with the weight of
200-250 g were prepared. The animals were kept under standard condition (12 h light/dark cycle, 20 to $22^{\circ} \mathrm{C}$ temperature) with food and water ad libitum.

\section{The study protocol}

The rats were randomly divided into four groups. In the control group (Co), the rats were treated with normal saline $(5 \mathrm{~mL} / \mathrm{kg}$; oral) for five consecutive weeks. In the GM group, the rats were administrated with normal saline ( $5 \mathrm{~mL} / \mathrm{kg}$; oral) for four consecutive weeks and then GM ( $80 \mathrm{mg} / \mathrm{kg} / \mathrm{d}$; intraperitoneal) for another one week. In the third (OB-200+GM) and fourth (OB-800+GM) groups, the rats were initially treated with hydro-alcoholic extract of $\mathrm{OB}(200 \mathrm{mg} / \mathrm{kg}$ and $800 \mathrm{mg} / \mathrm{kg}$ respectively; oral) for four consecutive weeks followed by intraperitoneal administration of $80 \mathrm{mg} / \mathrm{kg} / \mathrm{d}$ GM for another one week (Figure 1).

\section{Assessment of nephroprotective effects}

Blood and urine samples were obtained on the first day, 28 th day and at the end of study. Serum samples were collected by 15 minutes centrifugation of the blood sample at $3000 \mathrm{rpm}$. The serum levels of creatinine and blood urea nitrogen (BUN), Na, triglyceride and cholesterol, NF- $\mathrm{B}$ and tumor necrosis factor alpha (TNF- $\alpha$ ) were measured. The fractional excretion of sodium (FENa) was calculated according to the equation of $\mathrm{FeNa}=\frac{\mathrm{UNa} \times \mathrm{PCr}}{\mathrm{PNa} \times \mathrm{UCr}}$.

The rats were anesthetized by the intraperitoneal injections of ketamine and xylazine on day of 36th. Then, all the animals were slaughtered under complete anesthesia.

\section{Assessment of cellular apoptosis}

The terminal deoxynucleotidyl transferase-mediated dUTP nick end labeling (TUNEL) assay was used to

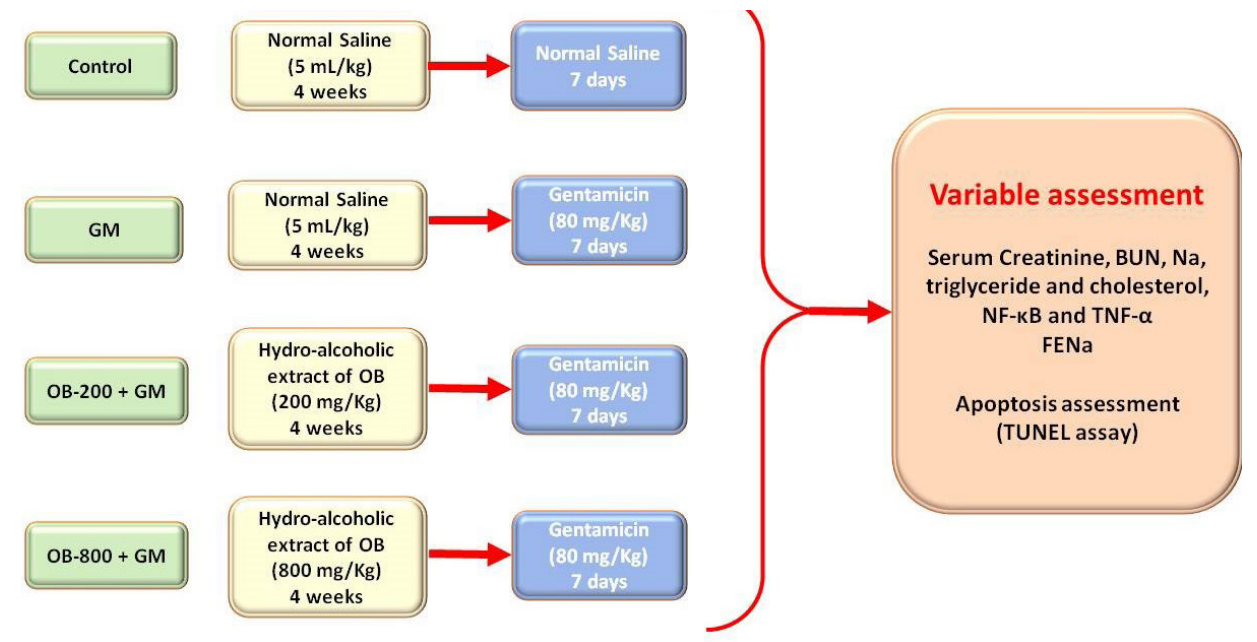

Figure 1. Study protocol and treatment groups. Wistar rats were categorized into 4 separate groups; control group was treated with normal saline for 4 weeks and then 7 days, Group 1 was treated by normal saline for 4 weeks followed by 7 days of $80 \mathrm{mg} / \mathrm{kg}$ gentamicin, Groups 2 and 3 were treated with 200 and $800 \mathrm{mg} / \mathrm{kg}$ Ocimum basilicum respectively for 4 weeks followed by 7 consecutive days of treatment with $80 \mathrm{mg} / \mathrm{kg}$ gentamicin. 
determine cellular apoptosis using a special kit (TUNEL; In Situ Cell Death Detection Kit, 11684817910 Roche). The total number of cells and the numbers of TUNEL positive cells (i.e. apoptotic cells) were enumerated using a fluorescence microscope.

\section{Ethical issues}

This experimental study was approved by the Ethics Committee of Shahid Beheshti University of Medical Sciences (\# IR.SBMU.RETECH.REC.1396.333) and was in accordance with the international guidelines for the care and use of laboratory animals.

\section{Statistical analysis}

The parametric variables were expressed using mean and standard deviation. The qualitative variables were presented using number and percentage. The data were analyzed by SPSS (v.24. IBM Inc. IL). For inferential analysis, chi-square test, one-way ANOVA, and Tukey's post hoc test were applied. The $P$ value $<0.05$ was considered as the statistical significance level.

\section{Results}

Overall, the histological and biochemical parameters of renal function significantly improved in mice treated with OB extract (OB-200+GM and OB-800+GM).

The level of urinary excretion of sodium was significantly higher in the GM group than other groups $(P<0.05$; Figure 2).

Also, a significant increase in serum BUN level in the GM group was seen. However, BUN initially increased and then decreased in the control, as well as the OB-200+GM and $\mathrm{OB}-800+\mathrm{GM}$ groups. We found a significant increase in serum BUN level in the GM group at the end of the study $(P=0.0001)$; however, such differences were not observed in other groups (Figure 3 ).

As shown in Figure 4, serum creatinine level significantly increased in the GM group, while no significant alternations were found in other groups. As shown in Figure 5, the FENa significantly increased in mice treated with GM alone (i.e. GM group). In contrast, significant reductions were

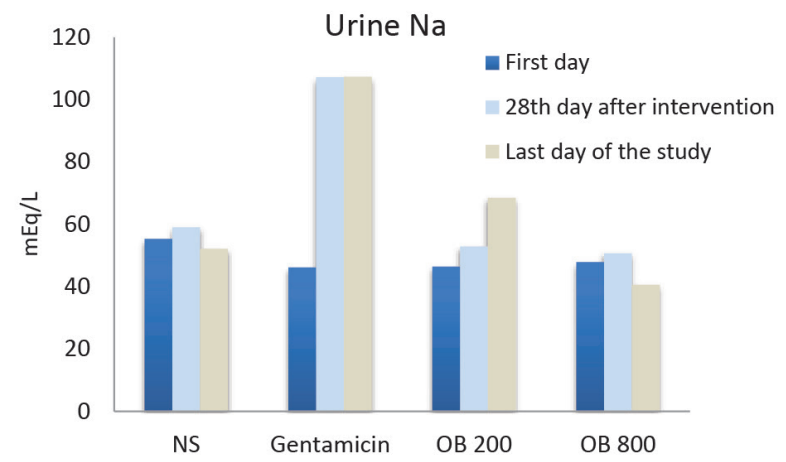

Figure 2. The alternations in urinary sodium excretion at different stages in different study groups.

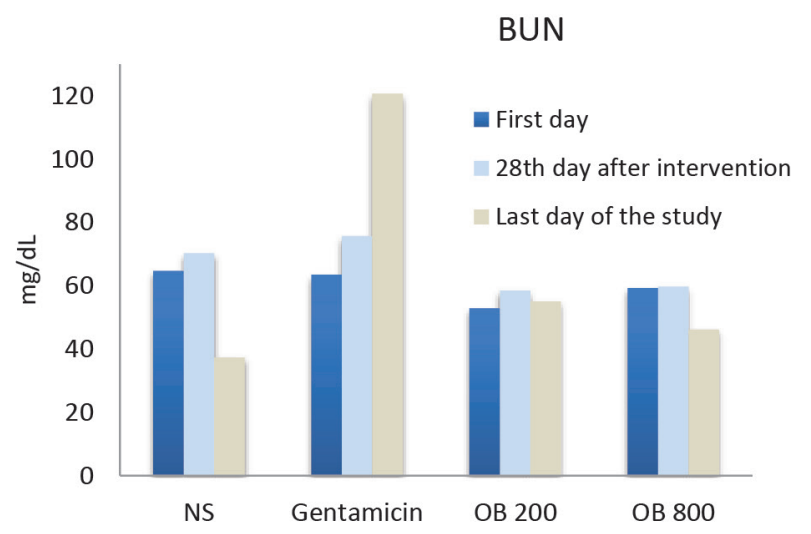

Figure 3. The alternations of serum BUN level at different stages in different study groups.

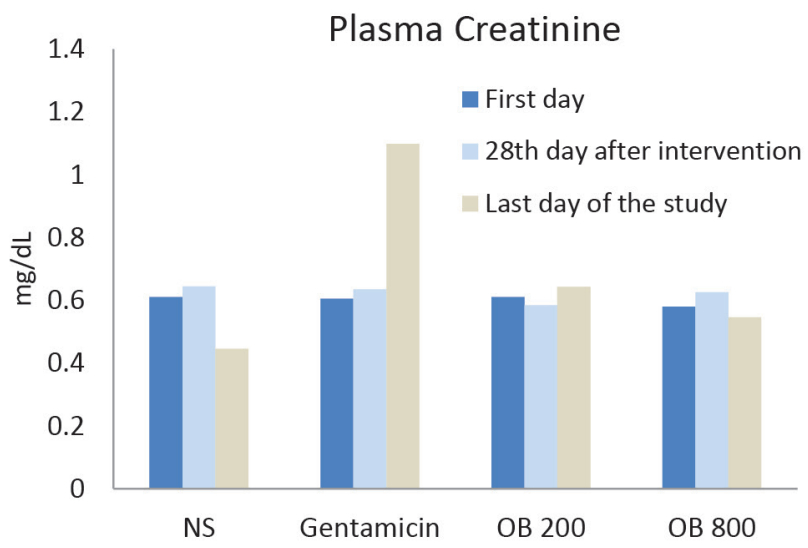

Figure 4. The alternations in serum creatinine level at different stages in different study groups.

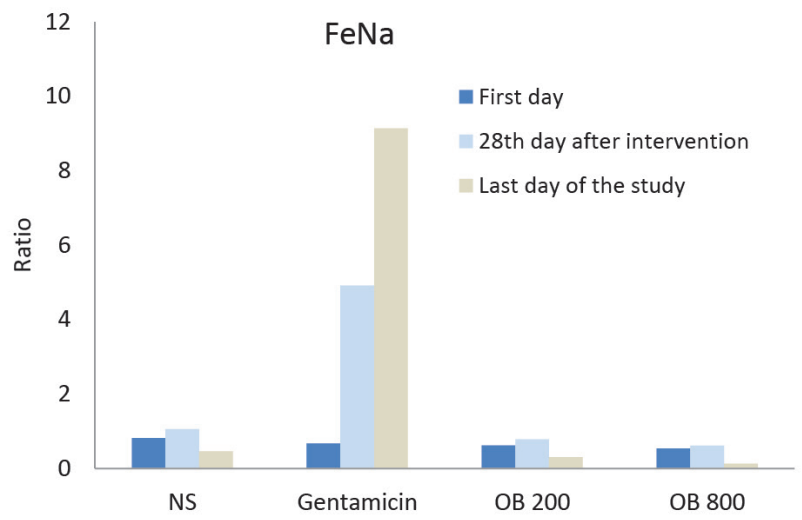

Figure 5. The levels of fractional excretion of sodium (FENa) at different stages in different study groups.

recorded in the levels of FENa in mice treated with either OB extract (i.e. OB-200+GM and OB-800+GM groups) or normal saline (i.e. control group). Overall, no statistically significant differences were observed in the levels of these biochemical parameters (urinary $\mathrm{Na}$ excretion, BUN, and FENa) among OB-200+GM, OB-800+GM, and control groups. 


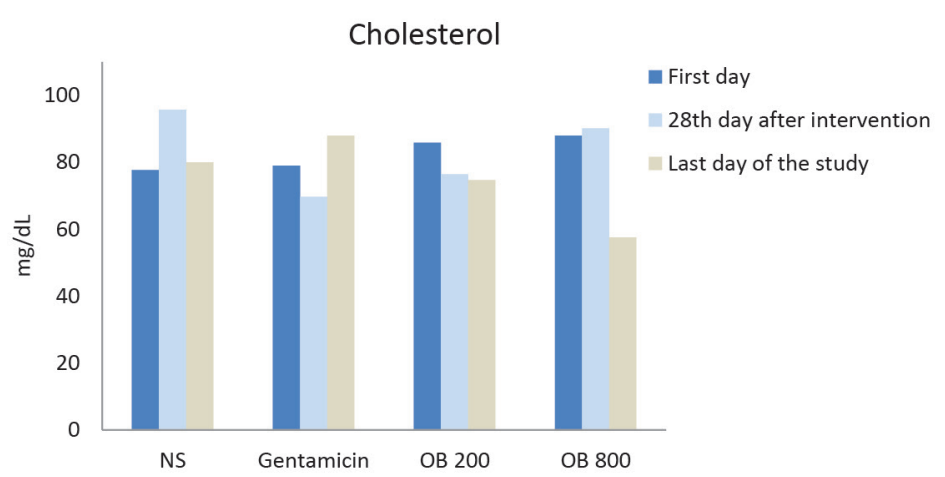

Figure 6. The levels of serum cholesterol at different stages and different study groups.

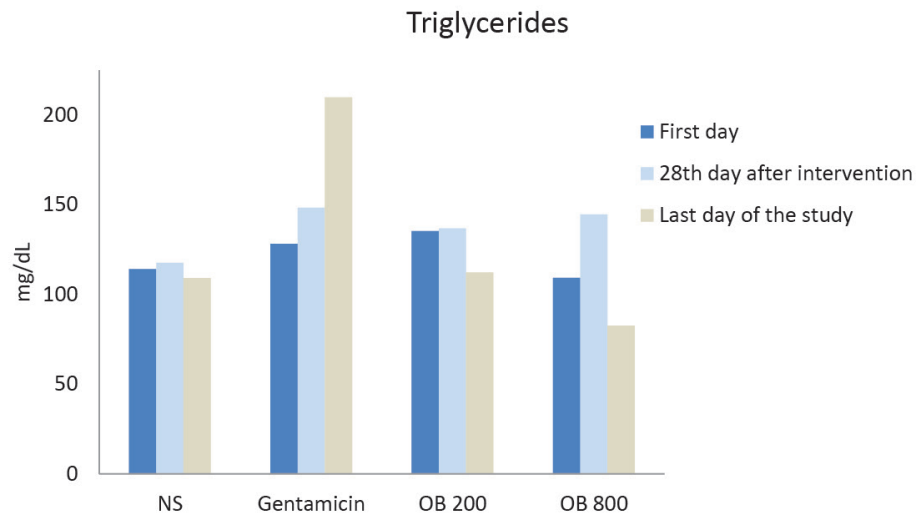

Figure 7. The serum levels of triglyceride at different stages in different study groups.

The serum cholesterol level decreased in mice treated with $200 \mathrm{mg} / \mathrm{kg}$ OB extract (OB-200+GM), while an initial increase and then a decrease in the serum cholesterol level in mice received $800 \mathrm{mg} / \mathrm{kg}$ OB extract (OB-800+GM) was detected (Figure 6). However, intra-groups comparison revealed no significant alternations in serum cholesterol levels during the study in neither of the groups.

A mild increase and then a slight decrease were reported in the serum level of triglyceride in the control group. In the OB-200+GM and OB-800+GM groups, relatively prominent decreases were observed in serum triglyceride level after an initial increase. This pattern of changes may indicate a delay effect for the herbal extract regimens. There was a significant difference in serum triglyceride level comparing the control with the OB-200+GM but not the OB-800+GM group. Additionally, a significant difference in serum triglyceride level comparing the OB$200+$ GM and OB-800+ GM groups was seen (Figure 7).

The mean levels of serum TNF- $\alpha$ were $81.0 \pm 1.5 \mathrm{pg} /$ $\mathrm{mL}, 67.25 \pm 1.43 \mathrm{pg} / \mathrm{mL}$ and $33.75 \pm 1.79 \mathrm{pg} / \mathrm{mL}$ in GM, OB-200+GM and OB-800+GM groups, respectively. Furthermore, the mean levels of NF- $\kappa \mathrm{B}$ were $77.75 \pm 1.03$ $\mathrm{pg} / \mathrm{mL}, 21 \pm 0.70 \mathrm{pg} / \mathrm{mL}$ and $9.75 \pm 1.10 \mathrm{pg} / \mathrm{mL}$ in $\mathrm{GM}, \mathrm{OB}-$ $200+\mathrm{GM}$ and $\mathrm{OB}-800+\mathrm{GM}$ groups, respectively. These observations indicated prominent increments in the levels of TNF- $\alpha$ and NF- $\kappa$ B followed by the administration of GM. However, pretreatment with $\mathrm{OB}$ extract prevented these elevations in a dose-dependent manner (i.e. lower TNF- $\alpha$, NF- $\kappa$ B levels in OB-800+GM than OB-200+GM group).

GM-induced-apoptosis in renal tubular cells was confirmed by the TUNEL assay. The ratio of TUNEL positive cells (i.e. apoptotic cells) significantly increased in the GM group compared with other groups. Pre-treatment with OB extract (i.e. OB-200+GM and OB-800+GM groups) diminished the rate of apoptosis in renal tubular cells. Accordingly, significant lower ratios of apoptotic cells were observed in the OB-200+GM and OB-800+GM groups compared to the GM group (Figures 8 and 9).

\section{Discussion}

Aminoglycoside-induced nephrotoxicity is characterized by decreased renal function and increased apoptotic renal cells. This phenomenon is one of the most prevalent and prominent adverse effects of aminoglycosides (12). GM-induced nephrotoxicity which is characterized by significant renal dysfunction has been associated with increased morbidity and mortality rates in animal models (13). The studies on animal models can help to better understand the possible mechanisms of GM- 

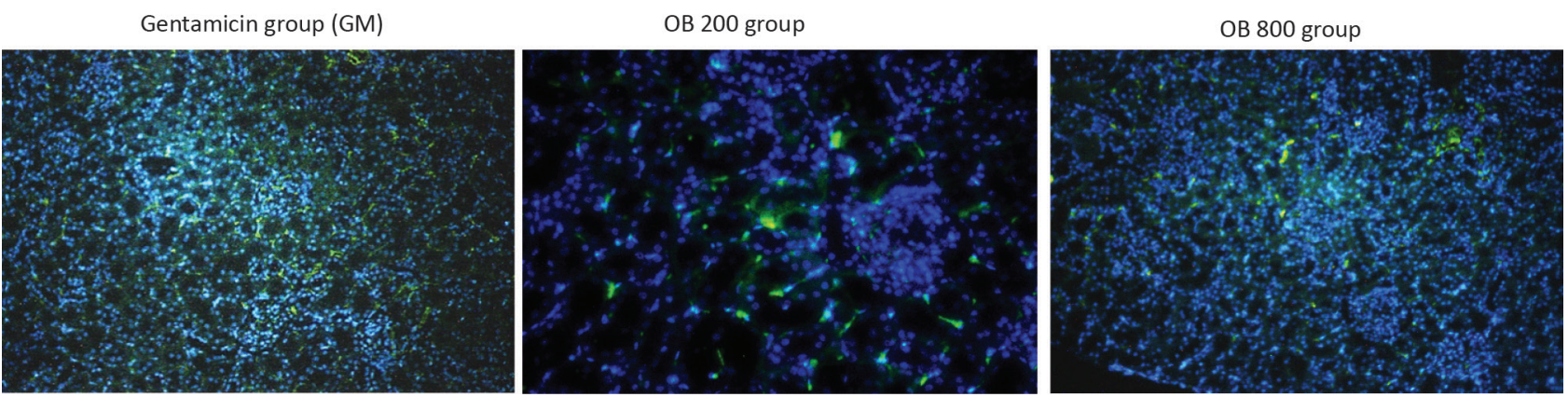

Figure 8. Effects of Ocimum basilicum extract against gentamicin-induced apoptosis in the gentamicin group, OB 200 group and OB 800 group by the TUNEL assay. Apoptotic cell nuclei were stained by the TUNEL assay with green, and all nuclei were stained by 4',6-diamidino2-phenylindole (DAPI) staining with blue (original magnification×100).

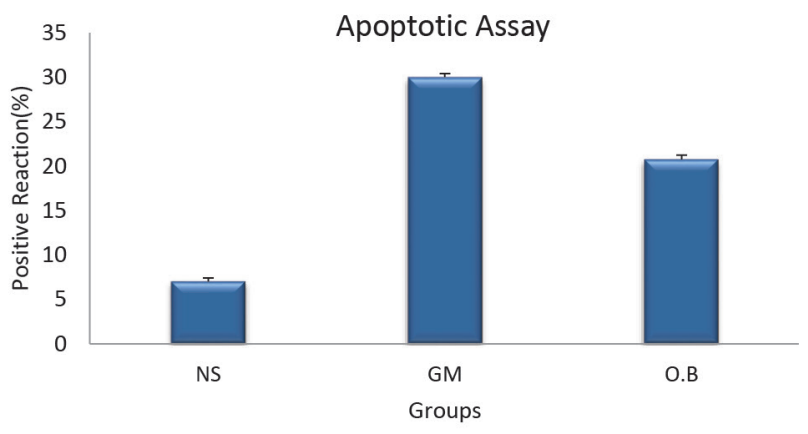

Figure 9. TUNEL-positive cells percentage was calculated.

induced nephrotoxicity and also to introduce appropriate chemical and herbal therapeutic agents (14). In our previous study, we reported that the administration of Portulaca oleracea extract decreased the risk of GMinduced nephrotoxicity by reducing apoptosis in renal cells and improving renal functional parameters (3). In the present study, we assessed the protective effects of OB hydro-alcoholic extract, as an herbal supplementary agent, against GM-induced nephrotoxicity. We observed that the extract of $\mathrm{OB}$ improved renal function and prevented apoptosis in renal tubular cells in the rat model of GM-induced nephrotoxicity. Our findings showed that the administration of GM in rats significantly increased the levels of urinary sodium excretion, as well as serum BUN and creatinine levels. However, pre-treatment with OB extract considerably decreased these biochemical parameters. We also found that the level of FENa, as one of the main renal functional parameters, was significantly lower in the rats treated with either normal saline or OBextract than those exposed to GM. Overall, no significant differences were observed between the two OB-treated groups with each other and with the control group in terms of these biochemical parameters and FENa level.

Apoptosis in renal tubular cells can be accompanied by prominent tubular necrosis and infiltration of inflammatory cells (15). Recently, Casanova et al highlighted the role of oxidative stress as a key mechanism involved in nephrotoxicity and also the effectiveness of antioxidant supplements in preserving renal function (16). We also indicated that GM administration significantly increased the levels of TNF- $\alpha$ and NF- $\mathrm{KB}$ inflammatory markers. Accordingly, OB extract significantly diminished the levels of these inflammatory markers in a dosedependent manner. In this regard, lower levels of TNF- $\alpha$ and NF- $\mathrm{KB}$ were detected in rats treated with $800 \mathrm{mg} /$ $\mathrm{kg}$ than those received $200 \mathrm{mg} / \mathrm{kg}$ OB extract. Our results suggested that $\mathrm{OB}$ extract can be administered as a potential anti-inflammatory and anti-oxidant herbal supplement for preventing GM-induced nephrotoxicity. Nevertheless, OB extract did not significantly affect serum lipid profile in rats treated with GM.

Aminoglycoside-induced apoptosis in renal tubular cells is one of the most important pathological characteristics of this type of drug-induced nephrotoxicity $(17,18)$. Apoptosis in renal epithelial cells has also been introduced as an important mechanism involved in renal hypoxic and chemical injuries $(17,18)$. In this study, we demonstrated that the ratio of TUNEL positive renal tubular cells (i.e. apoptotic cells) dramatically increased in rats treated with GM. On the other hand, pre-treatment with $\mathrm{OB}$ extract decreased the rate of apoptotic tubular cells in rats exposed to GM.

In a study by Oyouni et al on rats treated with tacrolimus, $\mathrm{OB}$ extract revealed both antioxidant and nephroprotective activities and also improved biochemical (BUN, creatinine, total protein and albumin) and histological parameters (19). The results of the recent study were consistent with our findings suggesting anti-oxidative and antiinflammatory roles for $\mathrm{OB}$ extract. On the other hand, Karaali et al have also asserted that OB extract may act as a natural antioxidant to prevent acetaminophen-induced acute liver and kidney toxicities (20).

\section{Conclusion}

In this study, we observed that $\mathrm{OB}$ extract induced antiapoptotic effects on renal tubular cells and also prevented pathological changes in GM-induced nephrotoxicity. Therefore, our findings provide comprehensive evidence 
supporting the beneficial role of $O$. basilicum, a traditional medicinal herb, as a potential therapeutic supplement to prevent the incidence and progression of renal impairment in GM-induced renal toxicity.

Authors' contribution

$\mathrm{HA}, \mathrm{FZ}, \mathrm{HHA}, \mathrm{SMH}$ and $\mathrm{AHA}$ participated in the conception of the study, data collection and analysis, and drafted the manuscript. HA, $\mathrm{FZ}, \mathrm{HHA}, \mathrm{SMH}$ and AHA contributed to drafting, critical reviewing, and finalization of the manuscript. All authors contributed to development of the manuscript and pproved the final manuscript.

Conflict of interests

The authors declare that they have no conflict of interest.

Ethical considerations

Ethical issues (including plagiarism, data fabrication, double publication) have been completely observed by the authors.

\section{Funding/Support}

None declared.

\section{References}

1. Makris K, Spanou L. Acute Kidney Injury: Definition, Pathophysiology and Clinical Phenotypes. Clin Biochem Rev. 2016; 37:85-98.

2. Elshiekh M, Kadkhodaee M, Seifi B, Ranjbaran M, Askari H. Up-regulation of nitric oxide synthases by erythropoietin alone or in conjunction with ischemic preconditioning in ischemia reperfusion injury of rat kidneys. General physiology and biophysics. 2017; 36:281-8. doi: 10.4149/gpb_2016058.

3. Sadidi M, Bakhtiyari M, Alirezaei A. Effects of the Portulaca oleracea extract on gentamicin-induced nephrotoxicity in male rats. Iran Red Crescent Med J. 2019;21:e83785. doi: 10.5812/ircmj.83785.

4. Sodimbaku V, Pujari L, Mullangi R, Marri S. Carrot (Daucus carota L.): Nephroprotective against gentamicin-induced nephrotoxicity in rats. Indian J Pharmacol. 2016;48:122-7. doi: 10.4103/0253-7613.178822

5. Randjelovic P, Veljkovic S, Stojiljkovic N, Sokolovic D, Ilic I. Gentamicin nephrotoxicity in animals: Current knowledge and future perspectives. EXCLI J. 2017;16:388-99. doi: 10.17179/ excli2017-165

6. Hun Choi K, II Kim T, Lim Chong D, Yung Lee H, Suk Han D. Gentamicin induced apoptosis of renal tubular epithelial (LLC-PK1) Cells. Korean J Intern Med. 2000;15:218-223. doi: 10.3904/kjim.2000.15.3.218.

7. Udupa V, Prakash V. Gentamicin induced acute renal damage and its evaluation using urinary biomarkers in rats. Toxicol Rep. 2019;6:91-99. doi: 10.1016/j.toxrep.2018.11.015

8. Benedec D, Parvu AE, Oniga I, Toiu A, Tiperciuc B. Effects of Ocimum basilicum L. extract on experimental acute inflammation. Rev Med Chir Soc Med Nat lasi. 2007; 111:1065-9.
9. Rodrigues LB, Martins A, Ribeiro-Filho J, Cesario F, FF EC, de Albuquerque TR, et al. Anti-inflammatory activity of the essential oil obtained from Ocimum basilicum complexed with beta-cyclodextrin (beta-CD) in mice. Food Chem Toxicol. 2017;109:836-846. doi: 10.1016/j.fct.2017.02.027.

10. Abd El-Ghffar EA, Al-Sayed E, Shehata SM, Eldahshan OA, Efferth T. The protective role of Ocimum basilicum L. (Basil) against aspirin-induced gastric ulcer in mice: Impact on oxidative stress, inflammation, motor deficits and anxietylike behavior. Food Funct. 2018;9:4457-4468. doi: 10.1039/ c8fo00538a.

11. Rashidian A, Roohi P, Mehrzadi S, Ghannadi AR, Minaiyan M. Protective Effect of Ocimum basilicum Essential Oil Against Acetic Acid-Induced Colitis in Rats. J Evid Based Complementary Altern Med. 2016;21:NP36-42. doi: 10.1177/2156587215616550.

12. Kaplan HM, Singirik E, Erdogan KE, Doran F. Protective effect of alpha-linolenic acid on gentamicin-induced ototoxicity in mice. Somatosens Mot Res. 2017;34:145-50. doi: 10.1080/08990220.2017.1356283.

13. Ansari MA, Raish M, Ahmad A, Ahmad SF, Mudassar S, Mohsin K, et al. Sinapic acid mitigates gentamicin-induced nephrotoxicity and associated oxidative/nitrosative stress, apoptosis, and inflammation in rats. Life Sci. 2016;165:1-8. doi: 10.1016/j.Ifs.2016.09.014.

14. Jablonski P, Howden BO, Rae DA, Birrell CS, Marshall VC, Tange J. An experimental model for assessment of renal recovery from warm ischemia. Transplantation. 1983;35:198204.

15. Ueda N, Shah SV. Tubular cell damage in acute renal failureapoptosis, necrosis, or both. Nephrol Dial Transplant. 2000; 15:318-23. doi:10.1093/ndt/15.3.318.

16. Casanova AG, Vicente-Vicente L, Hernandez-Sanchez MT, Pescador M, Prieto M, Martinez-Salgado C, et al. Key role of oxidative stress in animal models of aminoglycoside nephrotoxicity revealed by a systematic analysis of the antioxidant-to-nephroprotective correlation. Toxicology. 2017; 385:10-7. doi: 10.1016/j.tox.2017.04.015.

17. Savill J. Apoptosis and the kidney. J Am Soc Nephrol. 1994; 5:12-21.

18. Haghi-Aminjan $\mathrm{H}$, Asghari $\mathrm{MH}$, Goharbari $\mathrm{MH}$, Abdollahi $\mathrm{M}$. A systematic review on potential mechanisms of minocycline in kidney diseases. Pharmacol Rep. 2017;69:602-609. doi: 10.1016/j.pharep.2017.02.001.

19. Oyouni AAA, Saggu S, Tousson E, Rehman H. Immunosuppressant drug tacrolimus induced mitochondrial nephrotoxicity, modified PCNA and $\mathrm{BCl}-2$ expression attenuated by Ocimum basilicum L. in CD1 mice. Toxicol Rep. 2018;5:687-694. doi: 10.1016/j.toxrep.2018.06.003

20. Karaali HF, Fahmi RR, Borjac JM. Effect of Ocimum basilicum leaves extract on acetaminophen-induced nephrotoxicity in BALB/c mice. J Complement Integr Med. 2018. doi: 10.1515/ jcim-2018-0111. 